\title{
Nonlocal Integrable Partners to Generalized MKdV and Two-Dimensional Toda Lattice Equation in the Formalism of a Dressing Method with Quantized Spectral Parameter
}

\author{
A. Degasperis ${ }^{1}$, D. Lebedev ${ }^{2,4}$, M. Olshanetsky ${ }^{2}$, S. Pakuliak ${ }^{3}$, A. Perelomov ${ }^{2}$, \\ and P. Santini ${ }^{1}$ \\ 1 Dipartimento di Fisica, Università La Sapienza, P. le Aldo Moro 2, I-00185 Roma, and INFN, \\ Sezione di Roma, Italy \\ ${ }_{2}^{2}$ Institute for Theoretical and Experimental Physics, SU-117259 Moscow, USSR (permanent \\ address) \\ ${ }^{3}$ Institute for Theoretical Physics, SU-252130 Kiev, USSR \\ ${ }^{4}$ Max-Planck-Institut für Mathematik and Physikalisches Institut der Universität Bonn, \\ W-5300 Bonn 1, Federal Republic of Germany
}

Received December 4, 1990

\begin{abstract}
Two new hierarchies, MILW ${ }_{2}$ and a two-dimensional nonlocal Toda lattice are constructed. The characteristic property of the first one is the connection with the ILW ${ }_{2}$ hierarchy by means of $g l(2)$ Miura transformation. On the other hand, MILW ${ }_{2}$ equations turn out to be symmetry equations for a twodimensional nonlocal Toda lattice. A new version of the dressing technique with quantized spectral parameter is proposed.
\end{abstract}

\section{Introduction}

This paper is a direct continuation of our previous paper [1]. In [1] for the second representative $(n=2)$ of the Intermediate Long Waves $\left(\mathrm{ILW}_{n}\right)$ hierarchy [2], the zero-curvature representation with a noncommutative spectral parameter $\hat{\lambda}=\lambda e^{-2 i \hbar \partial_{x}}$ has been constructed. Here $\lambda$ and $\hbar$ are certain numerical parameters and $\partial_{x}=d / d x$. (The pioneering papers in the theory of the ILW equation are [3].) This has been achieved by means of a new version of the Zakharov-Shabat dressing technique with a noncommutative spectral parameter. Formally, the spectral parameter $\hat{\lambda}$ may be considered as a quantized spectral parameter $\lambda$, so that, in what follows we will refer to $\hat{\lambda}$ as a quantum spectral parameter.

It has also been explained in [1] that the $\mathrm{ILW}_{n}$ hierarchy appears as a special reduction of the KP hierarchy, which generalizes the well known reduction to $\mathrm{KdV}$-type equation. Therefore, this hierarchy of equations lies, roughly speaking, at the same level with $\mathrm{KdV}$ type equations. From the other side, one can imagine the $\mathrm{ILW}_{n}$ hierarchy as an one-parameter deformation of the $\mathrm{KdV}$ equation [3]. We will say in this case that ILW type equations are the nonlocal integrable partners to $\mathrm{KdV}$ type equations. 
There is a close connection of the two other hierarchies of equations, namely, MKdV and a two-dimensional Toda lattice, with KdV type equations [4-6]. As we prefer to look at the $\mathrm{ILW}_{n}$ and $\mathrm{KdV}_{n}$ hierarchies as different reductions of the same KP hierarchy, an attempt to find nonlocal integrable partners to $\mathrm{MKdV}$ and a two-dimensional Toda lattice are quite reasonable.

It turns out that this can be done using the ideas of [4] combined with the generalization of our dressing technique with quantum spectral parameter [1].

Let us state the main results of our paper.

The first result (see Sect. 2) is the construction of nonlocal integrable partners to the MKdV-type equation. One of the main properties of these equations is that these equations can be mapped to the ILW $\mathrm{IL}_{2}$-type equations with the help of the $g l(2)$ Miura transformation [4]. We will refer to this type of equations as MILW $_{2}$ equations.

In both cases of MILW ${ }_{2}$ and ILW 2 the situation is the following. We have the same phase space as in $\mathrm{KdV}$ or $\mathrm{MKdV}$ cases and Poisson brackets, but there are different possibilities to construct the Hamiltonians. One approach leads to the usual integrable $\mathrm{KdV}$ or MKdV-type equations. The other one leads to their nonlocal integrable partners. For the $\mathrm{ILW}_{n}$ case, this idea goes back to $[2,8]$.

The second result (see Sect. 3) is the generalization of the dressing method with the quantum spectral parameter constructed in our previous paper [1]. With the help of this technique we are constructing, in particular, the zero curvature representation for the $\mathrm{MILW}_{2}$-type equations.

The third result (see Sect. 4) is the construction of two-dimensional Toda lattice partners with the distinctive property: MILW $_{2}$ equations are the invariance equations to the last one. The crucial steps in proving this result are the special form of the zero-curvature representation, which, roughly speaking, corresponds to the analogous representation of the $\mathrm{MILW}_{2}$ equations and the application of some ideas of [4].

In Sect. 5, we explain why the simplest case of the $\operatorname{ILW}_{n}$ hierarchy when $n=1$ is not quite informative. Namely, in this situation the $\mathrm{ILW}_{1}$ and MILW ${ }_{1}$ equations

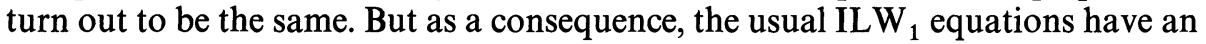
exceptional property. These are the symmetry equations for the nonlocal twodimensional Toda lattice equation [see Eq. (4.3) below].

In Sects. 6 and 7, Appendices A and B, we prove more technical results.

In this paper, as in [1], we restrict ourselves to the case $n=2$. The reason is that this is the first informative example in which we can imitate the suitable methods for dealing with different series of integrable nonlocal equations. The case of $n=1$ turns out to be exceptional (for details see Sect. 5) and we loose some important information. The general case is more complicated and will be considered in a separate publication [9].

\section{Part 1. Results, Motivations, and Examples}

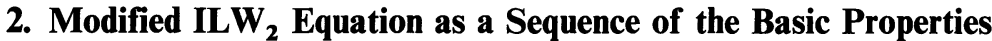 of Miura Transformation}

In this section we are introducing the new hierarchy of integrable equations, which plays the same role in the theory of ILW type equations as the MKdV equations in the theory of generalized $\mathrm{KdV}$ equations. In the $\mathrm{ILW}_{1}$ case, both of this equations 
coincide. It turns out that the first nontrivial case when these equations start to be different is the case of ILW 2 hierarchy.

1. Let us briefly summarize the results on the Hamiltonian properties of $\mathrm{KdV}$, MKdV-type equations and the Miura transformation that we need. (We will follow [4] in which a number of the important ideas of the previous papers are summarized [5-7].)

Let

$$
\mathscr{M}_{I}=\left\{\mathscr{L}=\partial_{x}-I+q \mid q=\left(\begin{array}{cc}
q_{11} & q_{12} \\
0 & q_{22}
\end{array}\right), I=\left(\begin{array}{ll}
0 & 0 \\
1 & 0
\end{array}\right)\right\}
$$

be the space of the first order differential operators with $2 \times 2$ matrix coefficients. The group $\tilde{N}=\left\{S \mid S=\left(\begin{array}{cc}1 & s(x) \\ 0 & 1\end{array}\right)\right\}$ acts on the space $\mathscr{M}_{I}$ by the gauge transformations:

$$
S^{-1}\left(\partial_{x}-I+q\right) S=\partial_{x}-I+\tilde{q} .
$$

Let $\overline{\mathscr{M}}_{I}$ be the factorspace $\mathscr{M}_{I} / \tilde{N}$. To define the Poisson bracket on the space of functionals on $\overline{\mathscr{M}}_{I}$ is the same as to define the Poisson bracket on the space $\mathscr{F}$ of gauge-invariant functionals on the linear space $\mathscr{M}_{I}$. This means that $\mathscr{F}$ is the space of the functionals satisfying the condition $f(q)=f(\tilde{q})$, where $\tilde{q}$ is defined by Eq. (2.2). There is a natural Poisson bracket on $\mathscr{F}$ :

$$
\{f, g\}=\int \operatorname{tr}\left(\operatorname{grad}_{q} f\left[\operatorname{grad}_{q} g, \partial_{x}+q-I\right]\right)
$$

where the $2 \times 2$ matrix $\operatorname{grad}_{q} f$ is determined by the following equation:

$$
\left.\frac{d}{d \varepsilon} f(q+\varepsilon h)\right|_{\varepsilon=0}=\int \operatorname{tr}\left(\operatorname{grad}_{q} f h\right) d x,
$$

with the matrix $h$ has the form $\left(\begin{array}{cc}h_{11} & h_{12} \\ 0 & h_{22}\end{array}\right)$.

Of course, the matrix $\operatorname{grad}_{q} f$ is defined by Eq. (2.4) up to adding an arbitrary uppertriangular matrix $\theta$ with zeros on the diagonal. But it is easy to check that the bracket (2.3) on the gauge invariant functionals does not depend on the choice of the $\operatorname{grad}_{q} f$, i.e. is correctly defined on the functionals from the space $\mathscr{F}$. It is also easy to see that $\{f, g\}(\tilde{q})=\{f, g\}(q)$, i.e. the Poisson bracket (2.4) is gauge invariant (for the proofs see [4]).

2. There exists a very useful realization of the space $\bar{M}_{I}$ as a linear space $\bar{M}_{I}$ of the second order differential operators with scalar coefficients. For any first-order differential operators $\mathscr{L}$ of the form $\left(\begin{array}{cc}\partial_{x}+q_{11} & q_{12} \\ -1 & \partial_{x}+q_{22}\end{array}\right)$ belonging to $\mathscr{M}_{I}$, one can construct the second-order differential operator $L$. This is given by the formula

$$
L=i(\mathscr{L}):=\partial_{x}^{2}+\left(q_{11}+q_{22}\right) \partial_{x}+q_{22}^{\prime}+q_{11} q_{22}+q_{12} .
$$

There exists a simple rule which we can use to obtain Eq. (2.5). Let us write down the equation $\mathscr{L} \psi=0$ to the vector function $\psi=\left(\psi_{1}, \psi_{2}\right)^{t}$. Exclude from this equation the function $\psi_{1}$. Then, for the function $\psi_{2}$ we obtain the equation $L \psi_{2}=0$, which defines the operator $L$ by the Eq. (2.5).

It is easy to check that if $i(\mathscr{L})=L$ then

$$
i\left(S^{-1} \mathscr{L} S\right)=L .
$$


This means that in this way the factorspace $\bar{M}_{I}$ can be realized as a linear space of the second order operators $\bar{M}_{I}$ with the unit coefficient before $\partial_{x}^{2}$.

To go from $\mathscr{M}_{I}$ to $\bar{M}_{I}$, it is necessary to fix the gauge. One can do this in the following way. Let $S=\left(\begin{array}{cc}1 & q_{22} \\ 0 & 1\end{array}\right)$, then

$$
S^{-1}\left(\partial_{x}-I+q\right) S=\partial_{x}-I+q^{\mathrm{can}},
$$

where $q^{\text {can }}=\left(\begin{array}{cc}u_{1} & u_{0} \\ 0 & 0\end{array}\right)$ and

$$
u_{1}=q_{11}+q_{22}, \quad u_{0}=q_{22}^{\prime}+q_{11} q_{22}+q_{12} .
$$

A useful example of the gauge invariant functionals on $\mathscr{M}_{I}$ is given by any functional depending on the variables $u_{1} u_{0}$, where $u_{i}$ is defined by Eq. (2.8). This is actually true owing to the fact that if $\tilde{q}$ is gauge equivalent to $q$, then $u_{1}$ and $u_{0}$, which are constructed by Eq. (2.8) from both $q$, and $\tilde{q}$ are the same.

3. There is another possibility to construct the phase space with a Poisson bracket different from (2.3). Namely, instead of going to the factorspace $\overline{\mathscr{M}}_{I}=\mathscr{M}_{I} / \widetilde{N}$, one can reduce $\mathscr{M}_{I}$ to $\mathscr{M}_{I}^{\text {red }}$, where ${ }^{1}$

$$
\mathscr{M}_{I}^{\text {red }}=\left\{\mathscr{L}^{\text {red }} \mid \mathscr{L}^{\text {red }}=\partial_{x}-I+q ; q=\operatorname{diag}\left(q_{1}, q_{2}\right)\right\} .
$$

There is the Poisson bracket on $\mathscr{M}_{I}^{\text {red }}$. Let $\psi, \varphi$ be the functionals depending on $q=\operatorname{diag}\left(q_{1}, q_{2}\right)$, then

$$
\{f, g\}_{\mathrm{r}}=\int \operatorname{tr}\left(\partial_{x}\left(\operatorname{grad}_{q} \varphi\right) \operatorname{grad}_{q} \psi\right) d x .
$$

In Eq. (2.10) the diagonal matrix $\operatorname{grad}_{q} \varphi$ is defined by the equation

$$
\left.\frac{d}{d \varepsilon} \psi(q+\varepsilon h)\right|_{\varepsilon=0}=\int \operatorname{tr}\left(\operatorname{grad}_{q} \psi h\right) d x,
$$

where $h$ has the form $h=\operatorname{diag}\left(h_{1}, h_{2}\right)$. It is clear, that now $\operatorname{grad}_{q} \psi$ is uniquely defined from Eq. (2.11).

If one starts from the operator $\mathscr{L}$ defined by the Eq. (2.1), then restricts it to $\mathscr{M}_{I}^{\text {red }}$ and finally maps this restriction onto the linear space $\bar{M}_{I}$ by means of $i$, then, as a result, one obtains the so-called Miura transformation $\mu: \mathscr{M}_{I}^{\text {red }} \rightarrow \bar{M}_{I}$, defined by the formula

$$
\left(\partial_{x}+q_{1}\right)\left(\partial_{x}+q_{2}\right)=\partial_{x}^{2}+u_{1} \partial_{x}+u_{0}
$$

here we have put $q_{11}=q_{1}$ and $q_{22}=q_{2}$.

A very important property of this transformation is the Hamiltonian property. More precisely, let $\bar{f}$ define the restriction of the functional $f \in \mathscr{F}$ to the space $\mathscr{M}_{I}^{\text {red }}$. Then there is

Important fact $[5,4]$. For any $f, g \in \mathscr{F}$

$$
\overline{\{f, g\}}=\{\bar{f}, \bar{g}\}_{\mathrm{r}} \text {. }
$$

The reduction (2.9) is not equivalent to a different fixing of the gauge, because one can easily find a nontrivial gauge transformation of the gauge group $\widetilde{N}$ which connects different choices of $q=\operatorname{diag}\left(q_{1}, q_{2}\right)$ 
Corollary. Let the functionals $H_{m}(q) \in \mathscr{F}$ be in involution with respect to the Poisson bracket $\{$,$\} , then their restrictions \bar{H}_{m}$ to the space $\mathscr{M}_{I}^{\text {red }}$ are in involution with respect to the Poisson bracket $\{,\}_{\mathrm{r}}$ on $\mathscr{M}_{I}^{\text {red }}$.

A typical example is given by the gauge invariant functionals $H_{m}(q)$ $=\int \operatorname{res} L^{m / 2} d x$ which are in involution with respect to the bracket $\{$,$\} . Therefore,$ $\bar{H}_{m}(q)=\int \operatorname{res}\left[\left(\partial_{x}+q_{1}\right)\left(\partial_{x}+q_{2}\right)\right]^{m / 2} d x$ are in involution with respect to the bracket $\{,\}_{\mathrm{r}}$ on $\mathscr{M}_{I}^{\text {red }}$. These are an infinite series of conservation laws for the $g l(2) \mathrm{KdV}$ and MKdV hierarchies, respectively.

4. Let us introduce the gauge invariant functionals of a special type on $\mathscr{M}_{I}$

$$
\tilde{\tau}_{X}(q)=\int \operatorname{res} L X d x
$$

where $X=\sum_{i=1}^{\infty} \partial_{x}^{-i} \circ X_{i}$ and $L$ is the second-order differential operator defined by Eq. (2.5). The Hamiltonian vector field with the Hamiltonian $\tilde{l}_{X}$, tangent to $\overline{\mathscr{M}}_{I}$ has the following form $[4,8]$ :

$$
q_{t}^{\text {can }}=\left[\operatorname{grad}_{q} \tilde{l}_{X}, \partial_{x}-I+q^{\text {can }}\right]
$$

where

$$
\operatorname{grad}_{q} \tau_{X}=\left[\begin{array}{cc}
X_{2} & X_{2}^{\prime}-X_{1}^{\prime \prime}+\left(u_{1} X_{1}\right)^{\prime}-u_{0} X_{1} \\
X_{1} & X_{2}-X_{1}^{\prime}+u_{1} X_{1}
\end{array}\right]
$$

Or, in the component form

$$
\left\{\begin{array}{l}
u_{1, t}=X_{1}^{\prime \prime}-\left(u_{1} X_{1}\right)^{\prime}-2 X_{2}^{\prime}, \\
u_{0, t}=X_{1}^{\prime \prime \prime}+2 u_{0} X_{1}^{\prime}+u_{0}^{\prime} X_{1}-u_{1}^{\prime \prime} X_{1}-2 u_{1}^{\prime} X_{1}^{\prime}-u_{1} u_{1}^{\prime} X_{1}-u_{1}^{2} X_{1}^{\prime}-u_{1} X_{2}^{\prime}-X_{2}^{\prime \prime} .
\end{array}\right.
$$

Now, restricting $\tilde{\tau}_{X}(q)$ on $\mathscr{M}_{I}^{\text {red }}$, one can obtain the Hamiltonian $\bar{\tau}_{X}(q)\left(q_{11}=q_{1}\right.$, $q_{22}=q_{2}$, and $q_{12}=0$ ) on $\mathscr{M}_{I}^{\text {red }}$. The Hamiltonian flow on $\mathscr{M}_{I}^{\text {red }}$ corresponding to this Hamiltonian with respect to the Poisson bracket $(2.10)$ has the form

$$
q_{t}=-\partial_{x} \operatorname{grad}_{q} \overline{\tilde{l}}_{X}, \quad q=\operatorname{diag}\left(q_{1}, q_{2}\right)
$$

where $\operatorname{grad}_{q} \overline{\bar{l}}_{X}$ is immediately calculated from Eq. (2.11):

$$
\operatorname{grad}_{q} \overline{\tilde{l}}_{X}=\left[\begin{array}{cc}
X_{2}+q_{2} X_{1} & 0 \\
0 & X_{2}-X_{1}^{\prime}+q_{1} X_{1}
\end{array}\right] .
$$

In the component form, Eq. (2.18) is the following:

$$
\left\{\begin{array}{l}
q_{1, t}=-\partial_{x}\left(X_{2}+q_{2} X_{1}\right) \\
q_{2, t}=-\partial_{x}\left(X_{2}-q_{1} X_{1}-X_{1}^{\prime}\right)
\end{array}\right.
$$

Direct computation shows that if $q_{1}, q_{2}$ are solutions of Eq. (2.20), then $u_{1}=q_{1}+q_{2}$ and $u_{0}=q_{2}^{\prime}+q_{1} q_{2}$ will be solutions of Eq. (2.17). This important property of the Miura transformation was discovered for the first time in the case of MKdV and $\mathrm{KdV}$ equations in [7] and later generalized to the $\mathrm{KdV}$-type equations in [4-6].

5. Now, we are in a position to define those types of equations which will be connected with the ILW 2 equations by means of the Miura transformation (2.12). Recall that to obtain an ILW 2 hierarchy, one needs to parametrize the coefficients $u_{1}, u_{0}$ of the operator $L$ by means of the formal series coefficients

$$
K\left(z, \partial_{z}\right)=1+\sum_{i \geqq 1} K_{i}(z) \partial_{z}^{-i}
$$


In this formula, the functions $K_{i}(z, t)$ are holomorphic, bounded and continuous up to the boundary in the strip $\Pi_{2 \hbar}=\{z \mid-2 \hbar<\operatorname{Im} z<0\}$.

More precisely, let us demand

$$
K^{-}\left(\partial_{x}\right) \partial_{x}^{2} K^{+}\left(\partial_{x}\right)^{-1}=\partial_{x}^{2}+u_{1} \partial_{x}+u_{0}=L,
$$

where $K_{ \pm}\left(\partial_{x}\right)=1+\sum_{i \geqq 1} K_{i}^{ \pm}(x, t) \partial_{x}^{-i}$, and $K_{i}^{-}(x)=K_{i}(x-2 i \hbar), K_{i}^{+}(x)=K_{i}(x){ }^{2}$

The constraint (2.22) gives expressions for the jumps $K_{i}^{-}-K_{i}^{+}$of the functions $K_{i}(z)$ and these boundary values of the $K_{i}(z)$ may be reconstructed by means of Sokhotsky-Plemely's formulas:

$$
\begin{gathered}
K^{-}(x)-K^{+}(x)=u(x), \\
K^{-}(x)+K^{+}(x)=-i T[u(x)],
\end{gathered}
$$

where $T[u]$ is an integral operator of the type

$$
T[u(x)]=\frac{1}{2 \hbar} \mathrm{P} . \mathrm{V} . \int_{-\infty}^{\infty} \operatorname{cth}(\pi(y-x) / 2 \hbar) u(y) d y .
$$

Now, expressing $X_{1}$ and $X_{2}$ through the coefficients of the formal series $K^{ \pm}\left(\partial_{x}\right)$ using the equation

$$
X=\sum_{i \geqq 1} \partial_{x}^{-i} \circ X_{i}=\left[K^{+}\left(\partial_{x}\right) \partial_{x}^{s-2} K^{-}\left(\partial_{x}\right)^{-1}\right]_{-}
$$

and substituting them into Eq. (2.17), we obtain the $\operatorname{ILW}_{2}$ hierarchy $[2,8,1]$. [In the right-hand side of Eq. (2.23), the lower index "-" means the projection onto the negative powers of $\partial_{x}$.]

To obtain the hierarchy of equations which are mapped onto the ILW equations by the Miura transformation (i.e. $\mathrm{MILW}_{2}$ hierarchy), one needs to put

$$
K^{-}\left(\partial_{x}\right) \partial_{x}^{2} K^{+}\left(\partial_{x}\right)^{-1}=\left(\partial_{x}+q_{1}\right)\left(\partial_{x}+q_{2}\right) \text {. }
$$

This gives the restriction on the coefficients $K_{i}$ in terms of $q_{i}$. Then one needs to define the functions $X_{1,2}$ by Eq. (2.23). Now $X_{1,2}$ will be expressed in terms of $q_{1,2}$ and it is necessary to substitute them to Eq. (2.20). This gives us the MILW 2 equation

Example. If $s=2$, then

$$
\begin{aligned}
& X_{1}=-u_{1}, \\
& X_{2}=u_{1}^{2}-u_{0}+i T\left[u_{1}^{\prime}\right],
\end{aligned}
$$

or in terms of $q_{1,2}$,

$$
\begin{aligned}
& X_{1}=-\left(q_{1}+q_{2}\right), \\
& X_{2}=\left(q_{1}+q_{2}\right)^{2}-\left(q_{2}^{\prime}+q_{2} q_{1}\right)+i T\left[q_{1}^{\prime}+q_{2}^{\prime}\right],
\end{aligned}
$$

then MILW 2 Eqs. (2.20), (2.23), and (2.24) will be the following:

$$
\begin{aligned}
& q_{1, t}=-\partial_{x}\left(q_{1}^{2}-q_{2}^{\prime}+i T\left[q_{1}^{\prime}+q_{2}^{\prime}\right]\right), \\
& q_{2, t}=-\partial_{x}\left(q_{2}^{2}+q_{1}^{\prime}+i T\left[q_{1}^{\prime}+q_{2}^{\prime}\right]\right) .
\end{aligned}
$$

These are exactly the equations which are mapped onto Eqs. (2.17) and (2.25) by the Miura transformation (2.12).

${ }^{2}$ Sometimes we will omit the sign " + " in $K_{i}^{+}$and $K^{+}\left(\partial_{x}\right)$ and write simply $K_{i}, K\left(\partial_{x}\right)$ 
6. From $[2,8]$ we know that there is an infinite series of conservation laws $H_{s}(q)$ of the ILW $\mathrm{IL}_{2}$-type equations which are in involution with respect to the Poisson bracket (2.3). The explicit formula can be obtained as follows. Let $K\left(\partial_{x}\right)$ satisfy Eq. (2.22). Then

$$
H_{s}(q) \sim \int_{-\infty}^{\infty} \operatorname{res}_{\lambda} \lambda^{s} \ln \frac{K^{+}(\lambda)}{K^{-}(\lambda)} d x
$$

where $K^{ \pm}(\lambda)=1+\sum_{i \geqq 1} K_{i}^{ \pm} \lambda^{-i}$

To calculate $\bar{H}_{s}$, we simply need to replace the condition (2.22) by the condition (2.24) and once more use Eq. (2.28). But now, Eq. (2.28) gives us an infinite series of the quantities $\bar{H}_{s}$ which, due to the property of the Miura's map, are in involution with respect to the Poisson bracket (2.10).

Since the Miura transformation maps the $\mathrm{MILW}_{2}$ equations onto the $\mathrm{ILW}_{2}$ ones, it follows that

$$
\bar{H}_{s, t}=0 \text {, }
$$

where $q_{1,2}$ are the solutions of (2.20), (2.23), and (2.24).

\section{Generalization of the Zakharov-Shabat Dressing Technique with Quantized Spectral Parameter}

In this section we are going to construct a generalization of the dressing technique with quantized spectral parameter which has been described in our previous paper [1]. The new version is available now both to $\mathrm{ILW}_{2}$ hierarchy and to MILW $\mathrm{M}_{2}$ one. It turns out that it is possible to dress the operator $\partial_{x}-\hat{\Lambda}$ up to the operator $\partial_{x}-\hat{\Lambda}+q$, where $q$ is an arbitrary upper triangular matrix.

1. Let us consider the "initial" spectral problem

$$
\left(\partial_{x}-\hat{\Lambda}\right) \dot{\psi}=0 \text {, }
$$

where

$$
\hat{\Lambda}=I+\hat{\lambda} e_{1,2} \equiv\left(\begin{array}{ll}
0 & \hat{\lambda} \\
1 & 0
\end{array}\right), \quad \hat{\lambda}=\lambda e^{-2 i \hbar \partial_{x}}
$$

The column $\stackrel{\leftrightarrow}{\psi}=\left(\stackrel{\circ}{\psi}_{1}, \stackrel{\circ}{\psi}_{2}\right)^{\mathrm{t}}$ is a solution of Eq. (3.1) and it follows from this equation that $\stackrel{\circ}{\psi}_{1}=\stackrel{\circ}{\psi}_{2, x}$.

Define the formal series in $\hat{A}$ by the equation

$$
G(\hat{\Lambda}) \equiv \sum_{i=0}^{\infty} G_{i}(x, t) \hat{\Lambda}^{-i}=\sum_{i=0}^{\infty}\left(\begin{array}{cc}
K_{1, i} & K_{2, i} \\
0 & K_{3, i}
\end{array}\right) \hat{\Lambda}^{-i},
$$

where $K_{i, j}=K_{i, j}(x, t)$ are some functions of $x$ and $t$.

Let

$$
\psi=G(\hat{\Lambda}) \stackrel{\varphi}{ } .
$$

Impose an additional condition on the coefficients $K_{i, j}$ :

$$
G(\hat{\Lambda})\left(\partial_{x}-\hat{\Lambda}\right) G^{-1}(\hat{\Lambda})=\partial_{x}-\hat{\Lambda}+q
$$


where now $q$ is not the same as Eq. (3.5) in [1], but

$$
q=\left(\begin{array}{cc}
q_{11} & q_{12} \\
0 & q_{22}
\end{array}\right) \text {. }
$$

If such $G(\hat{\Lambda})$ exists, then $\psi$ is defined by Eqs. (3.3) and (3.4) and will be a solution to the following spectral problem:

$$
\left(\partial_{x}-\hat{\Lambda}+q\right) \psi=0 .
$$

Note that Eq. (3.4) gives more general restrictions on the coefficients $K_{i, j}$ as compared to those we have in the case $q=q^{\mathrm{can}}=\left(\begin{array}{cc}u_{1} & u_{0} \\ 0 & 0\end{array}\right)$ in our previous paper [1]. In the case $q=q^{\text {can }}$, the prescription of a dressing with quantized spectral parameter was recalculated from the known case of scalar integro-differential equations [2].

The following lemma tells us that the condition (3.4) can be resolved:

Lemma 1. Fix $G(\hat{\Lambda})$ by Eqs. (3.2) and (3.4). Then the recursive equations on the coefficients $K_{i, j}$ which follow from $E q$. (3.4) can be solved and, moreover, let us define the formal integral operators

$$
K_{i}\left(\partial_{x}\right)=\sum_{j=0}^{\infty} K_{i, j} \partial_{x}^{-j}
$$

Then the conditions we are looking for can be written in the following form:

$$
\begin{gathered}
K_{1}\left(\partial_{x}\right) \text { be an arbitrary Volterra operator }\left(K_{1,0}=1\right), \\
K_{2}\left(\partial_{x}\right)=\left[K_{3}\left(\partial_{x}\right)-K_{1}\left(\partial_{x}\right)\right] \partial_{x}+K_{3}^{\prime}\left(\partial_{x}\right)+q_{22} K_{3}\left(\partial_{x}\right), \\
K_{3}^{-}\left(\partial_{x}\right) \partial_{x}^{2}=\left[\left(\partial_{x}+q_{11}\right)\left(\partial_{x}+q_{22}\right)+q_{12}\right] K_{3}\left(\partial_{x}\right) \quad\left(K_{3,0}=1\right) .
\end{gathered}
$$

Let us stress that it is necessary to find only the operator $K_{3}\left(\partial_{x}\right)$, then $K_{2}\left(\partial_{x}\right)$ is defined by $E q$. (3.6b) up to an arbitrary operator $K_{1}\left(\partial_{x}\right)$, so that all the information is concentrated in Eq. (3.6c).

See the proof in Appendix A.

2. Due to the Zakharov-Shabat ideology we need one more restriction on the functions $K_{i, j}(x, t)$, to construct the zero-curvature equations.

Lemma 2. Let us demand

$$
G(\hat{\Lambda})\left(\partial_{t_{s}}-\hat{\Lambda}^{s}\right) G^{-1}(\hat{\Lambda})=\partial_{t_{s}}-V(\hat{\Lambda}),
$$

where $V(\hat{\Lambda})=\sum_{i=0}^{s} V_{i}(x, t) \hat{\lambda}^{i}$. Equation (3.7) gives the same conditions to the time evolution of the elements $K_{i, j}(x, t)$ of the matrices $G_{i}(x, t)$ that follow from the equation

$$
K_{3, t_{s}}\left(\partial_{x}\right) K_{3}\left(\partial_{x}\right)^{-1}+\left(K_{3}\left(\partial_{x}\right) \partial_{x}^{s} K_{3}\left(\partial_{x}\right)^{-1}\right)_{-}=0,
$$

which can be solved recursively.

The proof is given in Appendix B. 
3. Now, let the formal matrix valued series $G(\hat{\Lambda})$ satisfy the conditions of the previous lemmas, then from

$$
\begin{aligned}
0 & =\left[\partial_{x}-\hat{\Lambda}, \partial_{t_{s}}-\hat{\Lambda}^{s}\right] \\
& =\left[G(\hat{\Lambda})\left(\partial_{x}-\hat{\Lambda}\right) G^{-1}(\hat{\Lambda}), G(\hat{\Lambda})\left(\partial_{t_{s}}-\hat{\Lambda}^{s}\right) G^{-1}(\hat{\Lambda})\right]
\end{aligned}
$$

it immediately follows that

$$
\left[\partial_{x}-U(\hat{\Lambda}), \partial_{t_{s}}-V(\hat{\Lambda})\right]=0,
$$

where the polynomial $V(\hat{\Lambda})$ is defined by Eq. (3.7) and $U(\hat{\Lambda})=\hat{\Lambda}-q$.

The standard arguments show that Eq. (3.10) is equivalent to the equation without quantum spectral parameter $\hat{\lambda}$ :

$$
q_{t}=\left[V_{0}, \partial_{x}-I+q\right] \text {. }
$$

Thus, we need exact information only about the structure of the matrix $V_{0}$. This is given by a nontrivial (due to the shift operator in the quantized spectral parameter $\hat{\lambda}$ ) theorem.

Theorem 1. Let $G(\hat{\Lambda})$ satisfy Eqs. (3.4) and (3.7), then

$$
\begin{aligned}
V_{0} & \equiv\left[G_{t_{s}}(\hat{\Lambda}) G^{-1}(\hat{\Lambda})+G(\hat{\Lambda}) \hat{\Lambda}^{s} G^{-1}(\hat{\Lambda})\right]_{0} \\
& =\left[\begin{array}{cc}
X_{2}+q_{22} X_{1} & X_{2}^{\prime}-X_{1}^{\prime \prime}+\left(q_{11} X_{1}\right)^{\prime}-q_{12} X_{1}+q_{22, t} \\
X_{1} & X_{2}-X_{1}^{\prime}+q_{11} X_{1}
\end{array}\right],
\end{aligned}
$$

where $X_{1}, X_{2}$ are defined from the equation

$$
X\left(\partial_{x}\right)=\sum_{i=1}^{\infty} \partial_{x}^{-i} \circ X_{i}:=\left[K_{3}\left(\partial_{x}\right) \partial_{x}^{s-2} K_{3}^{-}\left(\partial_{x}\right)^{-1}\right]_{-} .
$$

The proof of Theorem 1 can be found in Sect. 6 .

4. Now, there are two different possibilities. The first one is to fix the gauge as in Sect. 2. In the dressing technique language this means that $q_{11}=u_{1}, q_{12}=u_{0}$, $q_{22}=0$. Then, Eq. (3.12) will be the same as the equation on the $\operatorname{grad}_{q} \tau_{X}(2.16)$ and Eq. (3.11) turns into the ILW ${ }_{2}$ Eqs. (2.17), (2.22), (2.23), and (2.25).

The second possibility is to put

$$
q_{11}=q_{1}, \quad q_{22}=q_{2}, \quad q_{12}=0 .
$$

First of all Eq. (3.11) may be written in the coordinate form as follows:

$$
\left\{\begin{array}{l}
q_{11, t}=-V_{0,11}^{\prime}-q_{12} V_{0,21}-V_{0,12}, \\
q_{12, t}=-V_{0,12}^{\prime}+q_{12}\left(V_{0,11}-V_{0,22}\right)+\left(q_{22}-q_{11}\right) V_{0,12}, \\
q_{22, t}=-V_{0,22}^{\prime}+q_{12} V_{0,21}+V_{0,12} .
\end{array}\right.
$$

Here $V_{0, i j}$ defines the $i j$ element of the matrix $V_{0}$ [see Eq. (3.12)]. Now, put $q_{12}=0$. Then from the second equation in (3.15),

$$
V_{0,12}^{\prime}=\left(q_{22}-q_{11}\right) V_{0,12} \text {. }
$$

The simplest solution of the last equation is $V_{0,12}=0$. In this case, Eqs. (3.15) can be written in the form

$$
\begin{gathered}
q_{1, t}=-\partial_{x}\left(X_{2}+q_{2} X_{1}\right), \\
q_{2, t}=-\partial_{x}\left(X_{2}+q_{1} X_{1}-X_{1}^{\prime}\right) .
\end{gathered}
$$


This is exactly the MILW 2 equation (2.20). Note that the condition $V_{0,12}=0$ together with the equation $q_{12}=0$ is equivalent to Eq. (3.16b).

Conversely, if we are starting from the MILW 2 Eqs. (3.13) and (3.16), then Eq. (3.10) with the additional conditions (3.14), where in the $V_{0}$ term we are using once Eq. (3.16b), gives us a zero-curvature representation of the MILW equations.

\section{Nonlocal Partner to the Generalized Two-Dimensional Toda Lattice}

One of the important properties of a two-dimensional generalized Toda lattice is the invariance under the generalized MKdV equations [4, 5]. In this section we introduce nonlocal equations with the same invariance property under the generalized $\mathrm{MILW}_{2}$ equation.

1. Let us try to write a zero-curvature representation for the usual $g l(2)$ Toda lattice (see Eq. (10.4) in [4]) but with an important difference. Let us apply our prescription: write always $\lambda=\lambda e^{-2 i \hbar \partial_{x}}$ instead of $\lambda$ to obtain nonlocal integrable hierarchies; so let us define the operators

$$
\begin{aligned}
& \mathscr{L}=\partial_{x}-\hat{\Lambda}+\varphi^{\prime}, \\
& \overline{\mathscr{L}}=\partial_{\tau}-e^{-\varphi} \hat{\Lambda}^{-1} e^{\varphi},
\end{aligned}
$$

where

$$
\varphi=\left(\begin{array}{cc}
\varphi_{1} & 0 \\
0 & \varphi_{2}
\end{array}\right) \text { and } \varphi_{i}=\varphi_{i}(x, \tau), \quad \hat{\Lambda}=\left(\begin{array}{ll}
0 & \hat{\lambda} \\
1 & 0
\end{array}\right)
$$

Let us write the zero-curvature equation

$$
[\mathscr{L}, \overline{\mathscr{L}}]=0 .
$$

It is straightforward to check that Eq. (4.2) is equivalent to the following equation on the fields $\phi_{1}$ and $\phi_{2}$ :

$$
\left\{\begin{array}{l}
\phi_{1, x \tau}=e^{\phi_{1}(x, \tau, t)-\phi_{2}(x-2 i \hbar, \tau, t)}-e^{\phi_{2}(x, \tau, t)-\phi_{1}(x, \tau, t)} \\
\phi_{2, x \tau}=e^{\phi_{2}(x, \tau, t)-\phi_{1}(x, \tau, t)}-e^{\phi_{1}(x+2 i \hbar, \tau, t)-\phi_{2}(x, \tau, t)}
\end{array}\right.
$$

The fact that Eqs. (4.2) and (4.1) remains to be selfconsistent is not evident and there are no reasons to believe that our simple prescription will be successful. But it seems we have been lucky.

2. Of course, it is necessary to give some extra arguments to show that the Eqs. (4.2) and (4.3) are worth considering. One of such arguments is as follows.

Let $\varphi_{1}$ and $\varphi_{2}$ depend on the additional parameter $t$, and let $\mathscr{L}$ evolve in the $t$ direction as the $\mathrm{MILW}_{2}$ equation [we assume that $q=\varphi^{\prime}$ in Eqs. (2.27)]. Then there is an important fact:

Theorem 2. Let the operators $\mathscr{L}$ and $\overline{\mathscr{L}}$ be defined by Eqs. (4.1) and let $\mathscr{L}$ as an operator depending on the variable $t$ satisfy the MILW $W_{2}$ equation (2.27). Then

$$
\partial_{t}[\mathscr{L}, \overline{\mathscr{L}}]=0 .
$$

In other words, the MILW $W_{2}$ equations are the symmetry equations for Eq. (4.2).

3. This theorem is a consequence of a little bit stronger Theorem 3 . 
Theorem 3. Let the vector function $\psi=\psi(x, \tau, t)$ be a solution to the spectral problem

such that

$$
\left(\partial_{x}-\hat{\Lambda}+\varphi^{\prime}\right) \psi=0
$$

(i) The following system of linear equations is consistent:

$$
\begin{gathered}
\left(\partial_{x}-\hat{\Lambda}+\varphi^{\prime}\right) \psi=0, \\
\left(\partial_{\tau}-e^{-\varphi} \hat{\Lambda}^{-1} e^{\varphi}\right) \psi=0 .
\end{gathered}
$$

(Consistency condition is exactly Eq. (4.2).)

(ii) One more system of linear equations is consistent:

$$
\begin{gathered}
\left(\partial_{x}-\hat{\Lambda}+\varphi^{\prime}\right) \psi=0, \\
\left(\partial_{t}-V(\hat{\Lambda})\right) \psi=0,
\end{gathered}
$$

where $V(\hat{\Lambda})$ is defined by Eqs. (3.7) and (3.12) with $q=\varphi^{\prime}$. (Consistency condition is exactly the MIL $W_{2}$ equation with $q=\varphi^{\prime}$.)

Then the following equation is satisfied:

$$
\left[\partial_{\tau}-e^{-\varphi} \hat{\Lambda}^{-1} e^{\varphi}, \partial_{t}-V(\hat{\Lambda})\right]=0
$$

This means that all three equations are consistent:

$$
\left\{\begin{array}{l}
\left(\partial_{x}-\hat{\Lambda}+\varphi^{\prime}\right) \psi=0, \\
\left(\partial_{t}-V(\hat{\Lambda})\right) \psi=0, \\
\left(\partial_{\tau}-e^{-\varphi} \hat{\Lambda}^{-1} e^{\varphi}\right) \psi=0 .
\end{array}\right.
$$

The proof of Theorem 3 can be found in Sect. 7 and the proof of Theorem 2 follows immediately from Theorem 3. Really, Eq. (4.8) is the same as $[V(\hat{\Lambda}), \overline{\mathscr{L}}]=\overline{\mathscr{L}}_{t}$, so that

$$
\begin{aligned}
\partial_{t}[\mathscr{L}, \overline{\mathscr{L}}] & =[[V(\hat{\Lambda}), \mathscr{L}] \overline{\mathscr{L}}]+[\mathscr{L},[V(\hat{\Lambda}), \overline{\mathscr{L}}]] \\
& =[V(\hat{\Lambda}),[\mathscr{L}, \overline{\mathscr{L}}]]=0 .
\end{aligned}
$$

\section{The Exceptional ILW 1 Case}

In this section we will explain briefly which part of the results in the previous sections are lost and which remain unchanged for the simplest case of the ILW hierarchy.

The simplest representative of such an hierarchy is the equation

$$
u_{t}+2 u u_{x x}+i T\left[u_{x x}\right]=0 .
$$

The corresponding linear problem is

$$
\left\{\begin{array}{l}
\left(\partial_{x}+u\right) \psi^{+}=\lambda \psi^{-} \\
\psi_{t}^{ \pm}=M_{2}^{ \pm} \psi^{ \pm}
\end{array}\right.
$$

where $M_{2}^{ \pm}=\partial^{2}-2 K_{1, x}^{ \pm}$and $K_{1}^{ \pm}=(1 / 2)(\mp u-i T[u])$. In terms of the representation with a quantized spectral parameter, Eqs. (5.2) can be written as follows:

$$
\left\{\begin{array}{l}
\left(\partial_{x}+u-\hat{\lambda}\right) \psi^{+}=0 \\
{\left[\partial_{t}-\left(\hat{\lambda}^{2}-\hat{\lambda} u-u \hat{\lambda}+u^{2}+i T\left[u_{x}\right]\right)\right] \psi^{+}=0 .}
\end{array}\right.
$$


The corresponding dressing procedure can be formulated in the following manner. There exist formal series $G(\hat{\lambda})=1+\sum_{i=1}^{\infty} K_{i} \hat{\lambda}^{-i}$ such that

$$
\begin{gathered}
G(\hat{\lambda})\left(\partial_{x}-\hat{\lambda}\right) G^{-1}(\hat{\lambda})=\partial_{x}+u-\hat{\lambda}, \\
G(\hat{\lambda})\left(\partial_{t}-\hat{\lambda}^{2}\right) G^{-1}(\hat{\lambda})=\partial_{t}-V(\hat{\lambda}),
\end{gathered}
$$

where $V(\hat{\lambda})=\hat{\lambda}^{2}-\hat{\lambda} u-u \hat{\lambda}+u^{2}+i T\left[u_{x}\right]$. In terms of the coefficients of the formal Volterra operator $K\left(\partial_{x}\right)=1+\sum_{i \geqq 1} K_{i}(x) \partial_{x}^{-i}$ this gives the usual conditions on the coefficients $K_{i}(x)$ [2].

Looking at Eqs. (5.4), we observe the absence of the matrix structure of a zerocurvature representation and, consequently, in this case the MILW ${ }_{1}$ hierarchy coincides with the ILW ${ }_{1}$ one. The possibility to construct a nontrivial equation (5.1) appears due to the quantum spectral parameter in (5.4).

But it still remains possible to construct a nonlocal two-dimensional Toda type equation for which the ILW $\mathrm{I}_{1}$ equations will be symmetry equations. This strictly corresponds to the general ideology of Sect. 4. Really, let

$$
\begin{aligned}
& \mathscr{L}=\partial_{x}-\hat{\lambda}+\varphi^{\prime}, \\
& \overline{\mathscr{L}}=\partial_{\tau}-e^{-\varphi} \hat{\lambda}^{-1} e^{\varphi},
\end{aligned}
$$

where $\varphi=\varphi(x, \tau, t)$ is the scalar field. Then equation $[\mathscr{L}, \overline{\mathscr{L}}]=0$ is equivalent to the following equation on the field $\varphi$ (compare with the [10]),

$$
\phi_{x \tau}=e^{\phi(x+2 i \hbar, \tau)-\phi(x, \tau)}-e^{\phi(x, \tau)-\phi(x-2 i \hbar, \tau)} .
$$

The simplest symmetry equation is

$$
\phi_{t}+\left(\phi_{x}\right)^{2}+i T\left(\phi_{x x}\right)=0,
$$

which coincides with the ILW $\mathrm{IL}_{1}$ equation with $u=\varphi_{x}$. This reveals a new property of the ILW equation: it is a symmetry equation of the nonlocal one-component two-dimensional Toda lattice equation (5.6).

Putting $\hbar=0$ in Eq. (5.6) one can obtain a trivial equation

$$
\varphi_{x \tau}=0 \text {. }
$$

We would like to stress that Eqs. (5.5) and (5.6) do not coincide with the corresponding equations in [10] but are very similar to them.

\section{Part 2. Proofs}

\section{Proof of the Theorem 1}

For the proof of the theorem, we need the following technical result:

Lemma 3. Let $\psi=\left(\psi_{1}, \psi_{2}\right)^{t}$ be the solution of Eq. (3.5), then

$$
\begin{aligned}
\partial_{x}^{i} \psi_{2} & =O\left(\hat{\lambda}^{0}\right) \psi_{2}+O\left(\hat{\lambda}^{0}\right) \psi_{1}, \\
\partial_{x}^{-(i+1)} \psi_{2} & =O\left(\hat{\lambda}^{-1}\right) \psi_{2}+O\left(\hat{\lambda}^{-1}\right) \psi_{1}, \quad i \geqq 0,
\end{aligned}
$$


in particular

$$
\begin{aligned}
& \partial_{x}^{-1} \psi_{2}=\hat{\lambda}^{-1}\left(\psi_{1}+q_{11} \psi_{2}\right)+O\left(\hat{\lambda}^{-2}\right) \psi_{2}+O\left(\hat{\lambda}^{-2}\right) \psi_{1}, \\
& \partial_{x}^{-2} \psi_{2}=\hat{\lambda}^{-1} \psi_{2}+O\left(\hat{\lambda}^{-2}\right) \psi_{2}+O\left(\hat{\lambda}^{-2}\right) \psi_{1} .
\end{aligned}
$$

Proof. Let us prove for example the formula for $\partial_{x}^{-1} \psi_{2}$. As $\psi=G(\hat{\Lambda}) \dot{\psi}$, then due to $\partial_{x} \dot{\psi}=\hat{\Lambda} \dot{\psi}$ we obtain

$$
\begin{aligned}
\partial_{x}^{-1} \psi & =\partial_{x}^{-1} G(\hat{\Lambda}) \dot{\leftrightarrow} \\
& =\left(G(\hat{\Lambda}) \hat{\Lambda}^{-1}-G^{\prime}(\hat{\Lambda}) \hat{\Lambda}^{-2}+G^{\prime \prime}(\hat{\Lambda}) \hat{\Lambda}^{-3}+\ldots\right) G^{-1}(\hat{\Lambda}) \psi \equiv A \psi .
\end{aligned}
$$

We are interested in the last line in the matrix $A$, and more exactly those parts of the last line which have the first order in $\hat{\lambda}^{-1}$. The matrix $A$ satisfies the equation

$$
A G(\hat{\Lambda})=G(\hat{\Lambda}) \hat{\Lambda}^{-1}-G^{\prime}(\hat{\Lambda}) \hat{\Lambda}^{-2}+\ldots
$$

The series $G(\hat{\Lambda})$ has the form

$$
G(\hat{\Lambda})=\left(\begin{array}{cc}
1 & K_{2,0} \\
0 & 1
\end{array}\right)+\left(\begin{array}{cc}
K_{1,1} & K_{2,1} \\
0 & K_{3,1}
\end{array}\right) \hat{\Lambda}^{-1}+\ldots,
$$

where from Eq. (3.6b),

$$
K_{2,0}=K_{3,1}-K_{1,1}+q_{22} .
$$

Up to terms of the order greater than $\hat{\lambda}^{-1}$ the last line in Eq. (6.3) is equal to

$$
\left(\begin{array}{cc}
* & * \\
a_{21} & a_{21}\left(K_{2,0}+K_{1,1}\right)+a_{22}
\end{array}\right), \text { where } A=\left(\left(a_{i j}\right)\right) .
$$

The last line on the right-hand side of Eq. (6.3) is equal to

$$
\left(\begin{array}{cc}
* & * \\
\hat{\lambda}^{-1} & K_{3,1} \hat{\lambda}^{-1}
\end{array}\right)+\left(\begin{array}{cc}
* & * \\
O\left(\hat{\lambda}^{-2}\right) & O\left(\hat{\lambda}^{-2}\right)
\end{array}\right)
$$

so that $a_{21}=\hat{\lambda}^{-1}$, and

$$
a_{22}=\hat{\lambda}^{-1}\left(K_{3,1}^{-}-K_{3,1}-q_{22}\right)=\hat{\lambda}^{-1} q_{11} .
$$

As a result, we have

$$
\partial_{x}^{-1} \psi=\left(\begin{array}{cc}
* & * \\
\hat{\lambda}^{-1} & \hat{\lambda}^{-1} q_{11}
\end{array}\right) \psi+\ldots
$$

which is equivalent to the first of Eqs. (6.2). In the same manner one can prove all the statements of Lemma 3.

Now we are in a position to prove Theorem 1. We shall organize the proof as a series of direct calculations.

Step 1. We would like to calculate the zero coefficient in $\hat{\lambda}^{0}$ of the matrix

$$
V(\hat{\Lambda})=\left[G_{t_{s}}(\hat{\Lambda}) G^{-1}(\hat{\Lambda})+G(\hat{\Lambda}) \hat{\Lambda}^{s} G^{-1}(\hat{\Lambda})\right] .
$$

For this let us apply $V(\hat{\Lambda})$ to $\psi$, where $\psi$ is a solution of Eq. (3.5). From (3.5) it follows that $\psi_{1}=\psi_{2}^{\prime}$ and $L \psi_{2}=\hat{\lambda} \psi_{2}$. With the help of the Eqs. (3.1) and (3.3) one 
can rewrite

$$
\begin{aligned}
V(\hat{\Lambda}) \psi & =\left(G_{t_{s}}(\hat{\Lambda})+G(\hat{\Lambda}) \hat{\Lambda}^{s}\right) \stackrel{\psi}{\psi} \\
& =\left[\left(\begin{array}{cc}
K_{1, t}\left(\partial_{x}\right) & K_{2, t}\left(\partial_{x}\right) \\
0 & K_{3, t}\left(\partial_{x}\right)
\end{array}\right)+\left(\begin{array}{cc}
K_{1}\left(\partial_{x}\right) \partial_{x}^{s} & K_{2}\left(\partial_{x}\right) \partial_{x}^{s} \\
0 & K_{3}\left(\partial_{x}\right) \partial_{x}^{s}
\end{array}\right)\right] \dot{\psi}
\end{aligned}
$$

Step 2. From the Eqs. (3.3) and (3.1) it follows

$$
\psi_{2}=K_{3}\left(\partial_{x}\right) \dot{\psi}_{2} \text {. }
$$

So that the second line in Eq. (6.10) is equal to

$$
\left[K_{3, t}\left(\partial_{x}\right) K_{3}^{-1}\left(\partial_{x}\right)+K_{3}\left(\partial_{x}\right) \partial_{x}^{s} K_{3}^{-1}\left(\partial_{x}\right)\right] \psi_{2} \text {. }
$$

By Lemma $3 \partial_{x}^{-i} \psi_{2}=O\left(\hat{\lambda}^{-1}\right) \psi_{2}+O\left(\hat{\lambda}^{-1}\right) \psi_{1}$ when $i \geqq 1$. As we are interested in the zero term before $\psi_{2}$ in (6.11), the first term in this expression can be omitted. The last term in (6.11) can be rewritten as follows:

$$
K_{3}\left(\partial_{x}\right) \partial_{x}^{s} K_{3}^{-1}\left(\partial_{x}\right) \psi_{2}=\left(K_{3} \partial_{x}^{s-2}\left(K_{3}^{-}\right)^{-1} K_{3}^{-} \partial_{x}^{2} K_{3}^{-1}\right) \psi_{2}
$$

Let us recall that by the statement of the theorem, $K_{3}^{-} \partial_{x}^{2} K_{3}^{-1}=L$ and $L \psi_{2}=\hat{\lambda} \psi_{2}$. If one denotes by $W\left(\partial_{x}\right)$ and $X\left(\partial_{x}\right)$ the projection of the operator $K_{3} \partial_{x}^{s-2}\left(K_{3}^{-}\right)^{-1}$ on the positive and negative powers in $\partial_{x}$, then

$$
K_{3}\left(\partial_{x}\right) \partial_{x}^{s} K_{3}^{-1}\left(\partial_{x}\right) \psi_{2}=(W+X) \hat{\lambda} \psi_{2} \text {. }
$$

Once more we can omit the $W$ term on the right-hand side of Eq. (6.13) as long as we are interested in the zero in the $\hat{\lambda}$ term before $\psi_{2}$. Then the right-hand side of Eq. (6.13) is equal to

$$
\begin{aligned}
X L \psi_{2} & =\left(\partial_{x}^{-1} X_{1}+\partial_{x}^{-2} X_{2}+\ldots\right)\left(\partial_{x}^{2}+\left(q_{11}+q_{22}\right) \partial_{x}+q_{22}^{\prime}+q_{11} q_{22}+q_{12}\right) \psi_{2} \\
& =\left[X_{1} \partial_{x}+X_{2}-X_{1}^{\prime}+X_{1}\left(q_{11}+q_{22}\right)\right] \psi_{2}+\ldots
\end{aligned}
$$

But due to the equation $\partial_{x} \psi=(\hat{\Lambda}-q) \psi$ it follows that $\partial_{x} \psi_{2}=\psi_{1}-q_{22} \psi_{2}$ so that finally

$$
V_{0} \psi=\left(\begin{array}{cc}
* & * \\
X_{1} & X_{2}-X_{1}^{\prime}+q_{11} X_{1}
\end{array}\right) \psi
$$

We recall that $X=\sum_{i=1}^{\infty} \partial_{x}^{-i} \circ X_{i}=\left[K_{3} \partial_{x}^{s-2}\left(K_{3}^{-}\right)^{-1}\right]_{-}$.

Step 3. Now, let us calculate the upper line in Eq. (6.15). Due to Eq. (6.10), we have

$$
\left(K_{1, t}\left(\partial_{x}\right)+K_{1}\left(\partial_{x}\right) \partial_{x}^{s}\right) \dot{\circ}_{1}+\left(K_{2, t}\left(\partial_{x}\right)+K_{2}\left(\partial_{x}\right) \partial_{x}^{s}\right) \dot{\circ}_{2} .
$$

By Eqs. (3.3) and (3.1),

$$
\begin{aligned}
& \psi_{1}=K_{1}\left(\partial_{x}\right) \stackrel{\leftrightarrow}{\psi}_{1}+K_{2}\left(\partial_{x}\right) \stackrel{\circ}{2}_{2} \\
& \psi_{2}=K_{3}\left(\partial_{x}\right) \stackrel{\circ}{\psi}_{2}
\end{aligned}
$$

Thus,

$$
\begin{aligned}
& \stackrel{\circ}{\psi}_{1}=K_{1}^{-1}\left(\partial_{x}\right) \psi_{1}-K_{1}^{-1}\left(\partial_{x}\right) K_{2}\left(\partial_{x}\right) K_{3}^{-1}\left(\partial_{x}\right) \psi_{2}, \\
& \stackrel{\circ}{\psi}_{2}=K_{3}^{-1}\left(\partial_{x}\right) \psi_{2}
\end{aligned}
$$


and Eq. (6.16) will be written as follows

$$
\begin{aligned}
& \left(K_{1, t} K_{1}^{-1}+K_{1} \partial_{x}^{s} K_{1}^{-1}\right) \psi_{1} \\
& \left.\quad+\left(K_{2, t} K_{3}^{-1}+K_{2} \partial_{x}^{s} K_{3}^{-1}-K_{1, t} K_{1}^{-1} K_{2} K_{3}^{-1}-K_{1} \partial_{x}^{s} K_{1}^{-1} K_{2} K_{3}^{-1}\right) \psi_{2}\right) .
\end{aligned}
$$

But $\psi_{1}=\partial_{x} \psi_{2}+q_{22} \psi_{2}$ and $K_{2}\left(\partial_{x}\right)$ can be represented through the $K_{1}$ and $K_{3}$ by Eq. (3.6b). So, Eq. (6.19) has the form

$$
\left[\left(K_{3, t} K_{3}^{-1}+K_{3} \partial_{x}^{s} K_{3}^{-1}\right) \psi_{2}\right]^{\prime}+q_{22, t} \psi_{2}+q_{22}\left(K_{3, t} K_{3}^{-1}+K_{3} \partial_{x}^{s} K_{3}^{-1}\right) \psi_{2} .
$$

We would like to stress the remarkable cancellation of the operator $K_{1}\left(\partial_{x}\right)$ in Eq. (6.20). But the zero coefficient in $\hat{\lambda}$ standing before $\psi_{2}$ in the formula $\left(K_{3, t} K_{3}^{-1}+K_{3} \partial_{x}^{s} K_{3}^{-1}\right) \psi_{2}$ is equal to

$$
X_{1} \psi_{1}+\left(X_{2}-X_{1}^{\prime}+q_{11} X_{1}\right) \psi_{2} .
$$

Then, substituting Eq. (6.21) into Eq. (6.20), we will have

$$
\begin{aligned}
& X_{1}^{\prime} \psi_{1}+\left(X_{2}^{\prime}-X_{1}^{\prime \prime}+\left(q_{11} X_{1}\right)^{\prime}\right) \psi_{2}+X_{1} \psi_{1}^{\prime}+\left(X_{2}-X_{1}^{\prime}+q_{11} X_{1}\right) \psi_{2}^{\prime} \\
& \quad+q_{22, t} \psi_{2}+q_{22}\left(X_{1} \psi_{1}+\left(X_{2}-X_{1}^{\prime}+q_{11} X_{1}\right) \psi_{2}\right) .
\end{aligned}
$$

But, from equation $\partial_{x} \psi=(\hat{\Lambda}-q) \psi$ one has

$$
\begin{gathered}
\partial_{x} \psi_{1}=-q_{11} \psi_{1}-q_{12} \psi_{2}+\hat{\lambda} \psi_{2}, \\
\partial_{x} \psi_{2}=\psi_{1}-q_{22} \psi_{2} .
\end{gathered}
$$

Once more we need to omit the term $\hat{\lambda} \psi_{2}$ in Eq. (6.23a) and finally to substitute the result into Eq. (6.22). We thus have

$$
\left(X_{2}+q_{22} X_{1}\right) \psi_{1}+\left(q_{22, t}+X_{2}^{\prime}-X_{1}^{\prime \prime}-q_{12} X_{1}+\left(q_{11} X_{1}\right)^{\prime}\right) \psi_{2} \text {. }
$$

This reconstructs the upper line of the quantity $V_{0} \psi$, so that we have the result:

$$
V_{0} \psi=\left[\begin{array}{cc}
X_{2}+q_{22} X_{1} & X_{2}^{\prime}-X_{1}^{\prime \prime}+\left(q_{11} X_{1}\right)^{\prime}-q_{12} X_{1}+q_{22, t} \\
X_{1} & X_{2}-X_{1}^{\prime}+q_{11} X_{1}
\end{array}\right] \psi
$$

and $X=\sum \partial_{x}^{-i} \circ X_{i}=\left[K_{3} \partial_{x}^{s-2}\left(K_{3}^{-}\right)^{-1}\right]_{\text {. }}$. Thus we can easily conclude, from Eq. (6.25), the statement of the theorem.

\section{Proof of the Theorem 3}

Let $G(\hat{\Lambda})$ be the same as in Lemmas 1 and 2 . We remind that $\stackrel{\circ}{\psi}=G^{-1}(\hat{\Lambda}) \psi$. Then Eq. (4.2) is equivalent to the equation

$$
\left[\partial_{x}-\hat{\Lambda}, G^{-1}\left(\partial_{\tau}-e^{-\varphi} \hat{\Lambda}^{-1} e^{\varphi}\right) G\right]=0
$$

or

$$
\left[\partial_{x}-\hat{\Lambda}, \partial_{\tau}-v\right]=0
$$

where

$$
v=-G^{-1} \partial_{\tau} G-G^{-1}\left(e^{-\varphi} \hat{\Lambda}^{-1} e^{\varphi}\right) G .
$$


Equations (4.6) are equivalent to the following equations:

$$
\begin{gathered}
\left(\partial_{x}-\hat{\Lambda}\right) \stackrel{\psi}{\psi}=0, \\
\left(\partial_{\tau}-v\right) \dot{\psi}=0 .
\end{gathered}
$$

The same is true for Eqs. (4.7), which are equivalent to the following ones:

$$
\begin{aligned}
& \left(\partial_{x}-\hat{\Lambda}\right) \stackrel{\dot{\psi}}{=}=0, \\
& \left(\partial_{t}-\hat{\Lambda}^{s}\right) \dot{\psi}=0 .
\end{aligned}
$$

And finally (7.2) is the same that

$$
\partial_{x} v=[\hat{\Lambda}, v]
$$

Now, as $\partial_{x}, \partial_{\tau}$, and $\partial_{t}$ derivatives are commuting we have

$$
\begin{gathered}
\partial_{t} \partial_{\tau} \dot{\psi}=\partial_{t}(v \dot{\psi}) \\
\| \\
\partial_{\tau} \partial_{t} \dot{\psi}=\partial_{x}^{s}(v \stackrel{\leftrightarrow}{)},
\end{gathered}
$$

so that,

$$
v_{t} \dot{\psi}+v \hat{\Lambda}^{s} \dot{\psi}=\sum_{\alpha=0}^{s} C_{\alpha}^{s} v_{x}^{(\alpha)} \hat{\Lambda}^{s-\alpha} \dot{\psi} .
$$

Now, the statement of the theorem follows from Eq. (7.8) combined with

\section{Lemma 3.}

$$
\sum_{\alpha=0}^{s} C_{\alpha}^{s} v_{x}^{(\alpha)} \hat{\Lambda}^{s-\alpha} \dot{\psi}=\hat{\Lambda}^{s} v \dot{\psi} .
$$

Proof of the lemma. By Eq. (7.6) the right-hand side of Eq. (7.8) is equal to

$$
\sum_{\alpha=0}^{s} C_{\alpha}^{s} \operatorname{ad}_{\hat{\Lambda}}^{\alpha} v \hat{\Lambda}^{s-\alpha} \stackrel{\varphi}{ }
$$

where $\operatorname{ad}_{\hat{\Lambda}} v=[\hat{\Lambda}, v]$. One can rewrite expression $\operatorname{ad}_{\hat{\Lambda}}^{\alpha} v$ in (7.10) as

$$
\sum_{\beta=0}^{\alpha}(-1)^{\beta} C_{\beta}^{\alpha} \hat{\Lambda}^{\alpha-\beta} v \hat{\Lambda}^{\beta},
$$

so that, the right-hand side of Eq. (7.8) is equal to

$$
\begin{aligned}
\sum_{\alpha=0}^{s} C_{\alpha}^{s} \sum_{\beta=0}^{\alpha}(-1)^{\beta} C_{\beta}^{\alpha} \hat{\Lambda}^{\alpha-\beta} v \hat{\Lambda}^{s-(\alpha-\beta)} & =\sum_{\alpha=0}^{s} \sum_{\gamma=0}^{\alpha}(-1)^{\alpha-\gamma} C_{\alpha}^{s} C_{\alpha-\gamma}^{\alpha} \hat{\Lambda}^{\gamma} v \hat{\Lambda}^{s-\gamma} \\
& =\sum_{\gamma=0}^{s}\left[\sum_{\alpha=\gamma}^{s}(-1)^{\alpha-\gamma} C_{\alpha}^{s} C_{\alpha-\gamma}^{\alpha}\right] \hat{\Lambda}^{\gamma} v \hat{\Lambda}^{s-\gamma}
\end{aligned}
$$

where in the second equation we changed the index of summation $\beta=\alpha-\gamma$ and in the last one we changed the order of summation. But

$$
\begin{aligned}
\sum_{\alpha=\gamma}^{s}(-1)^{\alpha-\gamma} C_{\alpha}^{s} C_{\alpha-\gamma}^{\alpha} & =C_{\gamma}^{s} \sum_{\alpha=\gamma}^{s}(-1)^{\alpha-\gamma} C_{\alpha-\gamma}^{s-\gamma} \\
& =C_{\gamma}^{s} \sum_{\delta=0}^{s-\gamma}(-1)^{\delta} C_{\delta}^{s-\gamma}=\left\{\begin{array}{lll}
0, & \text { if } & \gamma<s, \\
1, & \text { if } & \gamma=s .
\end{array}\right.
\end{aligned}
$$


This completes the proof of Lemma 3 and prove the equation

$$
v_{t}=\left[\hat{\Lambda}^{s}, v\right] \text { or }\left[\partial_{t}-\hat{\Lambda}^{s}, \partial_{\tau}-v\right]=0 \text {. }
$$

The last equation is the same as

$$
\partial_{t} \overline{\mathscr{L}}=[V(\hat{\Lambda}), \overline{\mathscr{L}}]
$$

\section{Appendix A}

In this appendix we prove Lemma 1. Applying both sides of Eq. (3.4) to the solution $\psi$ of the equation

$$
\left(\partial_{x}-\hat{\Lambda}+q\right) \psi=0
$$

one can obtain

$$
G(\hat{\Lambda})\left(\partial_{x}-\hat{\Lambda}\right) \dot{\psi}=\left(\partial_{x}-\hat{\Lambda}+q\right) G(\hat{\Lambda}) \dot{\psi} .
$$

Here $\stackrel{\psi}{\psi}$ is a solution to Eq. (3.1) and $\psi$ is related to the $\stackrel{\circ}{\psi}$ by the equation

$$
\psi=G(\hat{\Lambda}) \stackrel{\varphi}{ }
$$

where $G(\hat{\Lambda})$ is defined by Eq. (3.2).

Step 1. Defining $K_{i}\left(\partial_{x}\right)=\sum_{j=0}^{\infty} K_{i, j} \partial_{x}^{-j}, i=1,2,3$ and using the equation $\stackrel{\circ}{\psi}_{1}=\stackrel{\circ}{\psi}_{2, x}$ and $\hat{\lambda} \dot{\psi}=\partial_{x}^{2} \dot{\psi}$, which follow from $\partial_{x} \dot{\psi}=\hat{\Lambda} \dot{\psi}$, one can rewrite Eq. (A.2) in the form

$$
\begin{aligned}
& {\left[\left(\begin{array}{ll}
K_{2}\left(\partial_{x}\right) & K_{1}\left(\partial_{x}\right) \partial_{x}^{2} \\
K_{3}\left(\partial_{x}\right) & 0
\end{array}\right)-\left(\begin{array}{cc}
0 & K_{3}^{-}\left(\partial_{x}\right) \partial_{x}^{2} \\
K_{1}\left(\partial_{x}\right) & K_{2}\left(\partial_{x}\right)
\end{array}\right)\right.} \\
& +\left(\begin{array}{cc}
q_{11} K_{1}\left(\partial_{x}\right) & q_{11} K_{2}\left(\partial_{x}\right)+q_{12} K_{3}\left(\partial_{x}\right) \\
0 & q_{22} K_{3}\left(\partial_{x}\right)
\end{array}\right) \\
& \left.+\left(\begin{array}{cc}
K_{1}^{\prime}\left(\partial_{x}\right) & K_{2}^{\prime}\left(\partial_{x}\right) \\
0 & K_{3}^{\prime}\left(\partial_{x}\right)
\end{array}\right)\right]\left(\begin{array}{c}
\stackrel{\leftrightarrow}{\psi}_{2, x} \\
\stackrel{\leftrightarrow}{\psi}_{2}
\end{array}\right)=0 \text {. }
\end{aligned}
$$

Or, equivalently,

$$
\left\{\begin{array}{l}
\left\{\left[K_{1}\left(\partial_{x}\right)-K_{3}^{-}\left(\partial_{x}\right)\right] \partial_{x}^{2}+\left[K_{2}\left(\partial_{x}\right)+K_{1}^{\prime}\left(\partial_{x}\right)+q_{11} K_{1}\left(\partial_{x}\right)\right] \partial_{x}\right. \\
\left.\quad+K_{2}^{\prime}\left(\partial_{x}\right)+q_{11} K_{2}\left(\partial_{x}\right)+q_{12} K_{3}\left(\partial_{x}\right)\right\} \dot{\vartheta}_{2}=0 \\
\left\{\left[K_{3}\left(\partial_{x}\right)-K_{1}\left(\partial_{x}\right)\right] \partial_{x}+K_{3}^{\prime}\left(\partial_{x}\right)-K_{2}\left(\partial_{x}\right)+q_{22} K_{3}\left(\partial_{x}\right)\right\} \stackrel{\circ}{\psi}_{2}=0
\end{array}\right.
$$

Step 2. Now, let us recall that $\dot{\varphi}_{2}$ is a solution of the equation $\partial_{x}^{2} \dot{\varphi}_{2}=\lambda \dot{\varphi}_{2}^{-}$. Therefore, it can be written as $\dot{\varphi}_{2}=e^{i \xi x+i c t}, \lambda=-\xi^{2} e^{-2 \hbar \xi}$. So that Eq. (A.5) are like $R\left(\partial_{x}\right) \dot{\varphi}_{2}=0$ or $R(i \xi) \dot{\varphi}_{2}=0$. Then the formal series $R(i \xi)=0$. In other words, all the coefficients of the series are equal to zero. But this means that $R\left(\partial_{x}\right)=0$. So, from Eq. (A.5), one can obtain the equations

$$
\left\{\begin{array}{l}
{\left[K_{1}\left(\partial_{x}\right)-K_{3}^{-}\left(\partial_{x}\right)\right] \partial_{x}^{2}+\left[K_{2}\left(\partial_{x}\right)+K_{1}^{\prime}\left(\partial_{x}\right)+q_{11} K_{1}\left(\partial_{x}\right)\right] \partial_{x}} \\
\quad+K_{2}^{\prime}\left(\partial_{x}\right)+q_{11} K_{2}\left(\partial_{x}\right)+q_{12} K_{3}\left(\partial_{x}\right)=0 \\
{\left[K_{3}\left(\partial_{x}\right)-K_{1}\left(\partial_{x}\right)\right] \partial_{x}+K_{3}^{\prime}\left(\partial_{x}\right)-K_{2}\left(\partial_{x}\right)+q_{22} K_{3}\left(\partial_{x}\right)=0}
\end{array}\right.
$$


Step 3. From the second of Eqs. (A.6), we obtain

$$
K_{2}\left(\partial_{x}\right)=\left[K_{3}\left(\partial_{x}\right)-K_{1}\left(\partial_{x}\right)\right] \partial_{x}+K_{3}^{\prime}\left(\partial_{x}\right)+q_{22} K_{3}\left(\partial_{x}\right)
$$

Substituting (A.7) into the first of Eqs. (A.6), it is easy to check that $K_{1}$ dependence is removed and the result will be a closer equation for the operator $K_{3}\left(\partial_{x}\right)$ :

$$
\begin{aligned}
& \left(K_{3}-K_{3}^{-}\right) \partial_{x}^{2}+\left(2 K_{3}^{\prime}+\left(q_{11}+q_{22}\right) K_{3}\right) \partial_{x} \\
& \quad+K_{3}^{\prime \prime}+\left(q_{11}+q_{22}\right) K_{3}^{\prime}+\left(q_{22}^{\prime}+q_{11} q_{22}+q_{12}\right) K_{3}=0 .
\end{aligned}
$$

But Eq. (A.8) is the same as

$$
K_{3}^{-} \partial_{x}^{2}=L K_{3},
$$

where $L=\left(\partial_{x}+q_{11}\right)\left(\partial_{x}+q_{22}\right)+q_{12}$.

Step 4. Let $K_{3}\left(\partial_{x}\right)=1+\sum_{i=1}^{\infty} K_{3, i} \partial_{x}^{-i}$, then Eq. (A.9) is equivalent to the recursive equations for the coefficients $K_{3, i}$ of the operator $K_{3}\left(\partial_{x}\right)$,

$$
\begin{aligned}
& u_{1}=K_{3,1}^{-}-K_{3,1}, \\
& u_{0}=K_{3,2}^{-}-K_{3,2}-2 K_{3,1}^{\prime}-u_{1} K_{3,1}, \\
& \cdots \\
& K_{3, i+2}^{-}-K_{3, i+2}=\left(K_{3, i}^{\prime \prime}+u_{1} K_{3, i}^{\prime}+u_{0} K_{3, i}\right)+2 K_{3, i+1}^{\prime}+u_{1} K_{3, i+1},
\end{aligned}
$$

where we put $u_{1}=q_{11}+q_{22}$ and $u_{0}=q_{22}^{\prime}+q_{11} q_{22}+q_{12}$. From the first equation one can reconstruct the function $K_{3,1}(z)$ which is holomorphic, bounded in the strip $\Pi_{2 \hbar}$ and continuous up to the boundary. This is achieved by using the integral operator

$$
K_{3,1}(z)=\frac{1}{4 i \hbar} \int_{-\infty}^{\infty} \operatorname{cth}(\pi(y-z) / 2 \hbar) u_{1}(y) d y
$$

Equation (A.11) reconstructs the function $K_{3,1}(z)$ with the fix jump

$$
K_{3,1}^{-}-K_{3,1}=u_{1}, \quad K_{3,1}^{-}+K_{3,1}=-i T\left[u_{1}\right] .
$$

So, for example, $K_{3,1}=\left(-i T\left[u_{1}\right]-u_{1}\right) / 2$. Of course, it is necessary to impose special analytical conditions on $u_{1}$ and $u_{0}$ such as smoothness and rapid vanishing at $\pm \infty$ together with some of its derivatives. Therefore, it is necessary to be careful at the next step, namely in the reconstruction of the coefficient $K_{3,2}$. Really, $K_{3,1} \sim(i / 4 \hbar) \operatorname{sign}(x) \int_{-\infty}^{\infty} u_{1}(y) d y$ as $|x| \rightarrow \infty$ because of the asymptotic behavior of $T[u(x)]$ at $|x| \rightarrow \infty$ :

$$
T[u(x)] \sim-\frac{1}{2 \hbar} \operatorname{sign}(x) \int_{-\infty}^{\infty} u(y) d y .
$$

But $\partial_{x} T[u]$ has the same vanishing properties at infinity as the function $u$, so that everything will be O.K. with $K_{3,1}^{\prime}$ and $K_{3,2}(z)$ can be reconstructed by Eq. (A.11) in the same manner, with the only difference that instead of $u_{1}$ one has to write $u_{0}+2 K_{1}^{\prime}+u_{1} K_{1}$. Looking at the general formula for $K_{3, i+2}^{-}-K_{3, i+2}$ in Eq. (A.10), one can prove by the same arguments that the right-hand side of the appropriate equation has appropriate analytical properties. Thus, the function $K_{3, i+2}(z)$ can be found from Eq. (A.11). This completes the proof. 


\section{Appendix B}

We must prove that

$$
\left[G_{t_{s}}(\hat{\Lambda}) G^{-1}(\hat{\Lambda})+G(\hat{\Lambda}) \hat{\Lambda}^{s} G^{-1}(\hat{\Lambda})\right]_{-}=0
$$

is equivalent to the following equation:

$$
K_{3, t_{s}}\left(\partial_{x}\right) K_{3}\left(\partial_{x}\right)^{-1}+\left(K_{3}\left(\partial_{x}\right) \partial_{x}^{s} K_{3}\left(\partial_{x}\right)^{-1}\right)_{-}=0,
$$

where, as before, the signs "-" in (B.1) and (B.2) mean the projections onto the negative powers of $\hat{\lambda}$ and $\partial_{x}$, respectively.

Using (6.11) and (6.20), one can see that the expression for $V(\hat{\Lambda}) \psi$ can be written as follows:

$$
V(\hat{\Lambda}) \psi=\left(\begin{array}{c}
{\left[\left(K_{3, t} K_{3}^{-1}+K_{3} \partial_{x}^{s} K_{3}^{-1}\right) \psi_{2}\right]^{\prime}+q_{22, t} \psi_{2}+q_{22}\left(K_{3, t} K_{3}^{-1}+K_{3} \partial_{x}^{s} K_{3}^{-1}\right) \psi_{2}} \\
\left(K_{3, t} K_{3}^{-1}+K_{3} \partial_{x}^{s} K_{3}^{-1}\right) \psi_{2}
\end{array}\right) .
$$

Then due to Lemma 3 , the absence of negative powers of $\hat{\lambda}$ in (B.3) means the absence of negative powers of $\partial_{x}$, so that using Eq. (B.3) we can conclude the statement of the lemma.

Acknowledgements. The authors are indebted to F. Calogero, A. Gerasimov, M. GolenishchevaKutuzova, and A. Reyman for stimulating discussions. We are also grateful to ITEP, Moscow and Instituto Nationale di Fisica Nucleare, Italy for financial support.

\section{References}

1. Degasperis, A. et al.: Generalized intermediate long-waves hierarchy in zero-curvature representation with noncommutative spectral parameter. Preprint of Max-Planck-Institute für Mathematik, Bonn, 1990

2. Lebedev, D., Radul, A.: Commun. Math. Phys. 91, 543 (1983)

3. Ablowitz, M., Kodama, Y., Satsuma, J.: Phys. Rev. Lett. 46, 677.(1981); Phys. Lett. A 73, 283 (1979); J. Math. Phys. 23 (4), 564 (1982)

4. Drinfeld, V., Sokolov, V.: J. Sov. Math. 30, 1975 (1984)

5. Kupershmidt, B., Wilson, G.: Invent. Math. 62, 403 (1981); Commun. Math. Phys. 81, 189 (1981)

6. Fordy, A., Gibbons, J.: Commun. Math. Phys. 77, 21 (1980); J. Math. Phys. 21, 2508 (1980); 22, 1170 (1981)

7. Miura, R.: J. Math. Phys. 9, 1202 (1968)

8. Lebedev, D., Pakuliak, S.: Comments on the Drinfeld-Sokolov Hamiltonian reduction. 1.g $l_{n}$ Case: Preprint GEF-Th-9/1990

9. Lebedev, D., Pakuliak, S.: To be published

10. Saveliev, M., Vershik, A.: Commun. Math. Phys. 126, 367 (1989); Phys. Lett. A 143, 121 (1990) 
